\title{
The Influence of Hippocampal Dopamine D2 Receptors on Episodic Memory Is Modulated by BDNF and KIBRA Polymorphisms
}

\author{
Goran Papenberg' ${ }^{1}$, Nina Karalija ${ }^{2}$, Alireza Salami ${ }^{1,2}$, Micael Andersson ${ }^{2}$, Jan Axelsson ${ }^{2}$, \\ Katrine Riklund $^{2}$, Ulman Lindenberger ${ }^{3,4}$, Lars Nyberg ${ }^{2}$, and Lars Bäckman ${ }^{1}$
}

\begin{abstract}
Episodic memory is a polygenic trait influenced by different molecular mechanisms. We used PET and a candidate gene approach to investigate how individual differences at the molecular level translate into between-person differences in episodic memory performance of elderly persons. Specifically, we examined the interactive effects between hippocampal dopamine D2 receptor (D2DR) availability and candidate genes relevant for hippocampus-related memory
\end{abstract}

\section{INTRODUCTION}

Episodic memory is influenced by a multitude of genes (Papassotiropoulos \& de Quervain, 2011; Rasch, Papassotiropoulos, \& de Quervain, 2010; de Quervain \& Papassotiropoulos, 2006) determined by different molecular mechanisms. Human aging is associated with a decline in episodic memory and with large interindividual differences in performance (Nyberg, Lövdén, Riklund, Lindenberger, \& Bäckman, 2012). By combining candidate gene and receptor imaging approaches, we investigate how individual differences at the molecular level may result in between-person differences in episodic memory performance among older individuals.

Nearly three decades ago, dopamine (DA) was shown to prolong long-term potentiation (LTP; Frey, Huang, \& Kandel, 1993; Frey, Schroeder, \& Matthies, 1990), a cellular mechanism necessary for successful memory formation and consolidation (for a review, see Cooke \& Bliss, 2006). Since then, animal studies have repeatedly shown that episodic memory performance is impaired when hippocampal DA receptors are blocked and enhanced when DA agonists are injected into the hippocampus (for a review, see Lisman \& Grace, 2005). In humans,

\footnotetext{
${ }^{1}$ Karolinska Institute and Stockholm University, ${ }^{2}$ Umeå University, ${ }^{3}$ Max Planck Institute for Human Development, Berlin, ${ }^{4}$ Max Planck UCL Centre for Computational Psychiatry and Ageing Research, Berlin and London
}

functioning. We show that the positive effects of high D2DR availability in the hippocampus on episodic memory are confined to carriers of advantageous genotypes of the brain-derived neurotrophic factor $(B D N F$, rs6265) and the kidney and brain expressed protein (KIBRA, rs17070145) polymorphisms. By contrast, these polymorphisms did not modulate the positive relationship between caudate D2DR availability and episodic memory. receptor imaging studies have related higher DA D2 receptor (D2DR) availability in the hippocampus to better episodic memory performance (Nyberg et al., 2016; Takahashi et al., 2007, 2008).

Two other proteins, the kidney and brain expressed protein (KIBRA) and the brain-derived neurotrophic factor (BDNF), have been implicated in hippocampal-based episodic memory. In the human brain, KIBRA is mainly expressed in the hippocampus and interacts with proteins involved in LTP (Schneider et al., 2010). BDNF promotes synaptic plasticity and is crucial for hippocampus-dependent learning and memory (Binder \& Scharfman, 2004). A genetic variation in the KIBRA gene has been associated with episodic memory, with T-allele carriers exhibiting higher performance than C-allele homozygotes (Papassotiropoulos et al., 2006). Similarly, a variation in the BDNF gene is associated with individual differences in secretion of this protein, which is greater in Val homozygotes than in Met carriers (Egan et al., 2003). Meta-analytic evidence confirms negative effects of the KIBRA C allele and the BDNF Met allele on human episodic memory (Kambeitz et al., 2012; Milnik et al., 2012). Using the Cognition, Brain, and Aging (COBRA) sample of healthy older adults $(n=181$, age $=$ 64-68 years), we investigate the interplay between hippocampal D2DR status and genetic variations in $B D N F$ and KIBRA and their contributions to episodic memory. Using the same data set, we have previously demonstrated that D2DR availability in caudate and hippocampus are associated with episodic memory performance (Nyberg et al., 2016). 
More specifically, we expect the hippocampal D2DRcognition link to be modulated by genes implicated in synaptic plasticity (i.e., $B D N F, K I B R A$ ). DA interacts with the same proteins involved in synaptic plasticity as KIBRA and BDNF, such as protein kinase Mzeta (PKMzeta), which is critical for maintenance of episodic memories (for a review, see Glanzman, 2013). BDNF facilitates LTP maintenance through PKMzeta (Mei, Nagappan, Ke, Sacktor, \& Lu, 2011), and KIBRA regulates learning and memory through stabilization of PKMzeta (Vogt-Eisele et al., 2014). DA release in the hippocampus enhances LTP (Lisman \& Grace, 2005). Importantly, however, PKMzeta is critical for the induction and maintenance of DA-induced LTP in the hippocampus (Navakkode, Sajikumar, Sacktor, \& Frey, 2010). Therefore, we hypothesize that individuals with high synaptic efficacy and plasticity (BDNF Val-allele, KIBRA T-allele) may benefit most from high hippocampal D2DR status and perform particularly well on episodic memory tasks. By contrast, we did not expect to find caudate D2DR availability to interact with variations in KIBRA and BDNF because of lower expression of these proteins in the striatum (Johannsen, Duning, Pavenstädt, Kremerskothen, \& Boeckers, 2008; Hofer, Pagliusi, Hohn, Leibrock, \& Barde, 1990).

\section{METHODS}

We have reported the COBRA study design, recruitment procedure, imaging protocols, and details of the cognitive and lifestyle battery (Nevalainen et al., 2015). COBRA is a multimodal imaging study of DA, brain structure and function, and cognition in normal aging. Here, we restrict the presentation to methodological details directly relevant to the present work. The study was approved by the local Ethics and Radiation Safety Committee of Umeå, Sweden, and all participants provided written informed consent before testing. Written consent was also acquired for storage of blood samples at the Department of Biobank Research at Norrland's University Hospital in Umeå, Sweden.

\section{Participants}

The initial sample included 181 healthy older individuals (64-68 years, mean $=66.2, S D=1.2 ; 81$ women) who were randomly selected from the population register of Umeå, a city in northern Sweden. Individuals with pathological deviations in brain and cognitive functions or circumstances that could bias task performance or obstruct imaging sessions (e.g., metal implants) were excluded. The resulting sample had lower prevalence of hypertension than nationwide reports (33\% in COBRA, 50\% nationwide; Carlsson, Wändell, de Faire, \& Hellénius, 2008), normal or slightly increased body mass index ( $>30$ in $14.4 \%$ of the sample), and $17.7 \%$ consumed nicotine. PET data were excluded for four individuals with imperfect segmentation of magnetic resonance (MR) images and PET-MR image coregistration and for one individual due to pathological deviations in the brain observed on the MR images. Genetic data were missing for one person. Thus, the effective sample included 175 individuals for the main analyses.

\section{MR and PET Imaging}

All participants underwent a 3-T MR (Discovery MR 750, GE Healthcare) and a PET scan (Discovery PET/CT 690, GE Healthcare) to obtain measures of structure and D2DR availability. A 3-D fast spoiled gradient-echo sequence was used to obtain high-resolution anatomical T1-weighted images. Imaging parameters were 176 sagittal slices, with slice thickness $=1 \mathrm{~mm}$, repetition time $=$ $8.2 \mathrm{msec}$, echo time $=3.2 \mathrm{msec}$, flip angle $=12^{\circ}$, and field of view $=25 \times 25 \mathrm{~cm}$.

PET was performed during resting-state conditions following an intravenous bolus injection of $250 \mathrm{MBq}\left[{ }^{11} \mathrm{C}\right]$ raclopride. Preceding the injection, a 5-min low-dose helical CT scan (20 mA, $120 \mathrm{kV}, 0.8 \mathrm{sec}$ per revolution) was obtained for PET attenuation correction. Following the bolus injection, a 55-min 18-dynamic PET scan was acquired (with $9 \times 120 \mathrm{sec}, 3 \times 180 \mathrm{sec}, 3 \times 260 \mathrm{sec}$, $3 \times 300 \mathrm{sec}$ frames). Attenuation- and decay-corrected PET images ( 47 slices, $15 \mathrm{~cm}$ field of view, $256 \times 256$-pixel transaxial images, voxel size $0.977 \times 0.977 \times 3.27 \mathrm{~mm}^{3}$ ) were reconstructed with the resolution recovery iterative VUE Point HD-SharpIR algorithm (GE Healthcare; Bettinardi et al., 2011; six iterations, 24 subsets, $3.0 \mathrm{~mm}$ postfiltering), yielding FWHM resolution of $3.2 \mathrm{~mm}$ (Wallstén, Axelsson, Sundström, Riklund, \& Larsson, 2013).

The D2DR status was determined by calculating the $\left[{ }^{11} \mathrm{C}\right]$ raclopride binding potential, $\mathrm{BP}_{\mathrm{ND}}$ (Innis et al., 2007; Logan et al., 1996; Mintun, Raichle, Kilbourn, Wooten, \& Welch, 1984). In brief, the PET emission scan format was converted from DICOM to NIfTI, corrected for head movements, and then coregistered to the corresponding T1 images using the Statistical Parametric Mapping software (SPM8; Ashburner et al., 2013). ROIs were delineated with the FreeSurfer 5.3 segmentation software (Han \& Fischl, 2007; Fischl et al., 2002, 2004). The cerebellar gray matter was used as a reference region due to negligible D2DR expression (Levey et al., 1993; Camps, Cortés, Gueye, Probst, \& Palacios, 1989; Farde, Hall, Ehrin, \& Sedvall, 1986). A time-activity curve, with the median of ROI voxel values from each time frame, was used to calculate $\mathrm{BP}_{\mathrm{ND}}$ using the Logan reference method (Logan et al., 1996), with linear regression from 18 to $55 \mathrm{~min}$. Left and right hemisphere $\mathrm{BP}_{\mathrm{ND}}$ values were averaged to give one value per participant and region. $\mathrm{BP}_{\mathrm{ND}}$ was calculated for hippocampus and caudate.

\section{Cognitive Measures}

The main cognitive domains examined offline in COBRA are episodic memory, working memory, and perceptual 
speed (see Nevalainen et al., 2015, for a detailed description). These domains were tested with three separate tasks each (a verbal, a numerical, and a figural task). For each task, summary scores were computed across the total number of blocks or trials. A summary score per ability was created by averaging the $T$-score measures $(M=50, S D=10)$ of each ability. Here, we restrict our description to the measure of episodic memory.

\section{Episodic Memory}

Word recall task. Participants were presented with 16 Swedish words (nouns) that appeared consecutively on the screen. The words were concrete and easy to spell, and all differed in the first three letters. During study, words were presented for $6 \mathrm{sec}$ each, with an ISI of $1 \mathrm{sec}$. After having seen the entire list of 16 items, participants reported the words they could recall by writing them down one-by-one in any order using a keyboard. Two test trials were administered $(\max =32)$.

Number-word task. This task consisted of memorizing pairs of two-digit numbers and concrete plural nouns (e.g., 46 dogs). During study, eight number-word pairs were displayed for 6 sec each, with an ISI of $1 \mathrm{sec}$. Following study, participants were requested to report, using the keyboard, the two-digit number associated with each noun shown on the screen (e.g., How many dogs?). During testing, words were presented one-by-one in a different order than during acquisition. If failing to recall the number, participants guessed. Two test trials were administered $(\max =16)$.

Object-position task. Participants were presented with a grid of $6 \times 6$ squares. Twelve objects were shown, one at a time, each at separate locations in the grid. Presentation time of each object-position pair was $8 \mathrm{sec}$, with an ISI of 1 sec. At test, all objects were shown adjacent to the grid, and the correct position of each object was reported by moving objects with the computer mouse (in any order) to the correct location in the grid. Again, participants were asked to guess for recall failures. Two test trials were performed $(\max =24)$.

\section{Genotyping}

In conjunction with the PET session, blood samples were collected from all participants and stored at the local biobank. Deoxyribonucleic acid (DNA) extraction and genotyping services were performed by LGC Genomics, using their in-house products (Hoddesdon, United Kingdom). In brief, DNA was extracted from the buffycoat fraction of blood samples using the Kleargene XL nucleic acid extraction kits, and genotyping was performed with KASPTM genotyping assays. In the genotyping analysis, the DNA template was mixed with a KASP master mix (containing KASP Taq polymerase, deoxynucleoside triphosphates, buffers, salts, two fluorescently labeled [FAM and HEX] reporter cassettes), and a single nucleotide polymorphism-specific KASP Assay mix (containing two allele-specific forward primers that differed at one base in the $3^{\prime}$-end and one common reverse primer). For analysis of the $B D N F$ polymorphism (rs6265), the primer sequence was $5^{\prime}$-GGC TGA CAC TTT CGA ACAC G/A-3' for the forward primers and 5'-GGT CCT CAT CCA ACA GCT CTT CTA T-3' for the reverse primer. For analysis of the KIBRA polymorphism (rs17070145), the primer sequence was 5'-C CTT GAT CCT GGA CCT C/ T-3' for the forward primers and $5^{\prime}$-CAG TAT AAA AGG AAA GCT CAG GAA CAG TT-3' for the reverse primer. Genotyping was carried out with polymerase chain reaction sessions, during which primers bound to their target sequences, reporter cassettes were incorporated into the DNA product, and amplification of the product was achieved. Allelic variants were determined via detection of FAM or HEX fluorescence for homozygotes or both for heterozygotes. DNA amplification failed for one sample.

The distributions of the KIBRA and $B D N F$ alleles were in Hardy-Weinberg equilibrium (KIBRA: T/T: $n=23$; C/T: $n=73 ; \mathrm{C} / \mathrm{C}: n=78 ; \chi^{2}<1, p>.1 ; B D N F:$ Met/Met: $n=$ 7; Met/Val: $n=49$; Val/Val: $n=119 ; \chi^{2}<1, p>.1$ ).

\section{Statistical Analyses}

Behavioral and demographic data were analyzed using SPSS for Windows 15 . We conducted linear regression analyses to investigate main and interactive effects between $\left[{ }^{11} \mathrm{C}\right]$ raclopride $\mathrm{BP}_{\mathrm{ND}}$ in the hippocampus and a genetic score based on KIBRA and BDNF on episodic memory. The same approach was performed for the caudate. There is no evidence that one of these polymorphisms would have a stronger effect on episodic memory than the other (for a review, see Papenberg, Lindenberger, \& Bäckman, 2015), and there were only a few carriers of two disadvantageous genotypes $(n=$ 22). To increase predictive power and enhance individual differences in synaptic efficacy and plasticity, we contrasted individuals carrying two advantageous genotypes (BDNF Val/Nal and KIBRA T-allele carriers; $n=61$ ) against the rest $(n=114)$. For illustrative purposes and further analyses, participants were grouped into three groups based on their D2DR availability status. Groups were based on a tertile split of the sample distribution of $\mathrm{BP}_{\mathrm{ND}}$ in the hippocampus (mean of left and right hemisphere). Reported mean differences stem from an ANCOVA, with D2DR group (low, average, high) and Gene score (no or one beneficial genotype, two beneficial genotypes) as between-subject factors and Episodic memory as the outcome. All analyses were adjusted for the DRD2 C957T polymorphism (rs6277), which affects $\left[{ }^{11} \mathrm{C}\right]$ raclopride affinity (Hirvonen et al., 2009) and thereby may inflate $\mathrm{BP}_{\mathrm{ND}}$ in individuals with high affinity (Karalija et al., 2019). Given the well-established female superiority in episodic memory (Maitland, Herlitz, Nyberg, Bäckman, 
\& Nilsson, 2004; Herlitz, Nilsson, \& Bäckman, 1997), sex was included as covariate. Despite the narrow age range, chronological age was added as covariate, given significant age differences among gene score groups $(p=.05$; Table 1).

The outlier labeling rule was used to identify outliers; the following formulas were computed to calculate the upper and lower limits, respectively, for outliers: Q3 + $(2.2 \times(\mathrm{Q} 3-\mathrm{Q} 1))$ and $\mathrm{Q} 1-(2.2 \times(\mathrm{Q} 3-\mathrm{Q} 1))$, where Q1 is the lower quartile (25th percentile of the data) and Q3 is the upper quartile (75th percentile). The number 2.2 is the value of the tuning parameter $g$, which was set to 2.2 following recommendations (Hoaglin \& Iglewicz, 1987). Multivariate outliers within and across groups were determined using Mahalanobi's distance, with the recommended $p<.001$ threshold for the $x^{2}$ value (Tabachnick $\&$ Fidell, 2007). There were no univariate or multivariate outliers. For all analyses, the alpha level was set to $p<.05$. Effect sizes are indicated by partial eta squared.

\section{RESULTS}

$\mathrm{BP}_{\mathrm{ND}}$ in the hippocampus was directly related to episodic memory performance: $\beta=.186, t(169)=2.492, p=$ .014 . By contrast, the gene score alone did not predict episodic memory, $\beta=.031, t(169)=0.412, p=.681$, which was also the case for KIBRA, $\beta=-.030, t(168)=-.426$, $p=.670$, and $B D N F, \beta=.056, t(169)=0.737, p=.462$. However, the regression analyses revealed a significant linear interaction between hippocampal $\mathrm{BP}_{\mathrm{ND}}$ and the gene score grouping in predicting episodic memory, $\beta=.842, t(167)=2.056, p=.041$. Adding the interaction term as a predictor resulted in a nonsignificant association for the hippocampus-memory link, $\beta=.055$, $t(167)=0.563, p=.574$. Despite a positive association

Table 1. Demographic, Health, and Lifestyle Information as a Function of Grouping

\begin{tabular}{|c|c|c|c|c|c|c|}
\hline \multirow[b]{3}{*}{ Beneficial Genotypes } & \multicolumn{2}{|c|}{ Low D2DR $B P_{N D}$} & \multicolumn{2}{|c|}{ Average $D 2 D R B P_{N D}$} & \multicolumn{2}{|c|}{ High $D 2 D R B P_{N D}$} \\
\hline & No/One & Two & No/One & Two & No/One & Two \\
\hline & $n=41$ & $n=17$ & $n=36$ & $n=24$ & $n=37$ & $n=20$ \\
\hline Age, years $(M \pm S D)$ & $66.2 \pm 1.1$ & $66.2 \pm 1.2$ & $65.9 \pm 1.2$ & $66.3 \pm 1.3$ & $66.0 \pm 1.3$ & $66.7 \pm 1.2^{\mathrm{a}}$ \\
\hline Women, \% & 48.8 & 47.1 & 47.2 & 58.3 & 37.8 & $30.0^{\mathrm{b}}$ \\
\hline Education, years $(M \pm S D)$ & $14.1 \pm 4.1$ & $12.93 \pm 2.7$ & $13.0 \pm 3.4$ & $12.0 \pm 3.4$ & $12.7 \pm 3.7$ & $14.9 \pm 2.9^{c}$ \\
\hline Retired, \% & 68.3 & 82.4 & 63.9 & 83.3 & 78.4 & $65.0^{\mathrm{b}}$ \\
\hline Body mass index $(M \pm S D)$ & $25.6 \pm 2.7$ & $25.6 \pm 3.4$ & $26.3 \pm 3.5$ & $26.0 \pm 3.5$ & $26.5 \pm 4.3$ & $27.0 \pm 4.5^{\mathrm{d}}$ \\
\hline Nicotine use, \% & 19.5 & 17.6 & 11.1 & 16.7 & 21.6 & $20.0^{\mathrm{b}}$ \\
\hline Systolic blood pressure $(M \pm S D)$ & $141.2 \pm 15.2$ & $142.8 \pm 12.9$ & $141.4 \pm 19.0$ & $140.0 \pm 20.7$ & $140.6 \pm 18.1$ & $145.3 \pm 18.7^{\mathrm{e}}$ \\
\hline Diastolic blood pressure $(M \pm S D)$ & $86.5 \pm 9.7$ & $84.7 \pm 9.9$ & $85.1 \pm 8.4$ & $82.5 \pm 11.8$ & $85.7 \pm 9.0$ & $85.15 \pm 11.3^{\mathrm{e}}$ \\
\hline Blood pressure medication, \% & 29.3 & 41.2 & 41.7 & 20.8 & 35.1 & $35.0^{\mathrm{b}}$ \\
\hline Hyperlipidemia medication, \% & 17.1 & 11.8 & 16.7 & 8.3 & 18.9 & $25.0^{\mathrm{b}}$ \\
\hline Cardiovascular medication, \% & 34.1 & 47.1 & 47.2 & 33.3 & 40.5 & $45.0^{\mathrm{b}}$ \\
\hline Depression/anxiety medication, \% & 2.4 & 0 & 11.1 & 12.5 & 8.1 & $0^{\mathrm{b}}$ \\
\hline Hours intellectual activity $(M \pm S D)$ & $34.4 \pm 18.5$ & $36.1 \pm 17.1$ & $32.5 \pm 16.1$ & $32.7 \pm 13.8$ & $35.7 \pm 14.4$ & $40.1 \pm 18.7^{\mathrm{e}}$ \\
\hline Hours physical activity $(M \pm S D)$ & $23.5 \pm 13.5$ & $21.7 \pm 13.7$ & $21.4 \pm 9.1$ & $21.8 \pm 12.0$ & $22.7 \pm 12.6$ & $20.3 \pm 12.0^{\mathrm{e}}$ \\
\hline Hours social activity $(M \pm S D)$ & $30.1 \pm 16.5$ & $36.4 \pm 23.4$ & $32.9 \pm 14.5$ & $35.1 \pm 19.6$ & $32.6 \pm 14.4$ & $29.5 \pm 18.7^{\mathrm{e}}$ \\
\hline
\end{tabular}

Self-reported leisure activities were assessed using a questionnaire that included 43 activities, 18 intellectual, 15 physical, and 10 social. Participants were asked to indicate for how many hours $(1,2,3, \ldots, 15+\mathrm{hr})$ they engage in each of the activities during a typical summer week. A sum score (hr/week) was computed across all intellectual, physical, and social activities, respectively. $M=$ mean; $S D=$ standard deviation.

${ }^{\text {a }}$ Trend for gene score group: $F(1,174)=3.9, p=.05$, with carriers of two beneficial genotypes being older.

${ }^{\mathrm{b}}$ Chi-square test $=$ not significant.

${ }^{c}$ Univariate ANOVA: Hc-Group $\times$ Gene Score Group: $F(1,174)=5.9, p=.02$, indicated the highest educational attainment for carriers of two beneficial genotypes and high D2DR availability.

${ }^{\mathrm{d}}$ Main effect for Hc-Group: $F(1,174)=4.3, p=.04$, indicating the highest body mass index in the group with high D2DR BP ${ }_{\mathrm{ND}}$.

${ }^{\mathrm{e}}$ Univariate ANOVA $=$ not significant. 
Figure 1. Episodic memory performance as a function of gene score grouping (no/one beneficial genotype, two beneficial genotypes) and hippocampal D2DR availability (low, average, high). Mean episodic memory performance for the sample $=50$. Errors bars represent 1 standard error around the means.

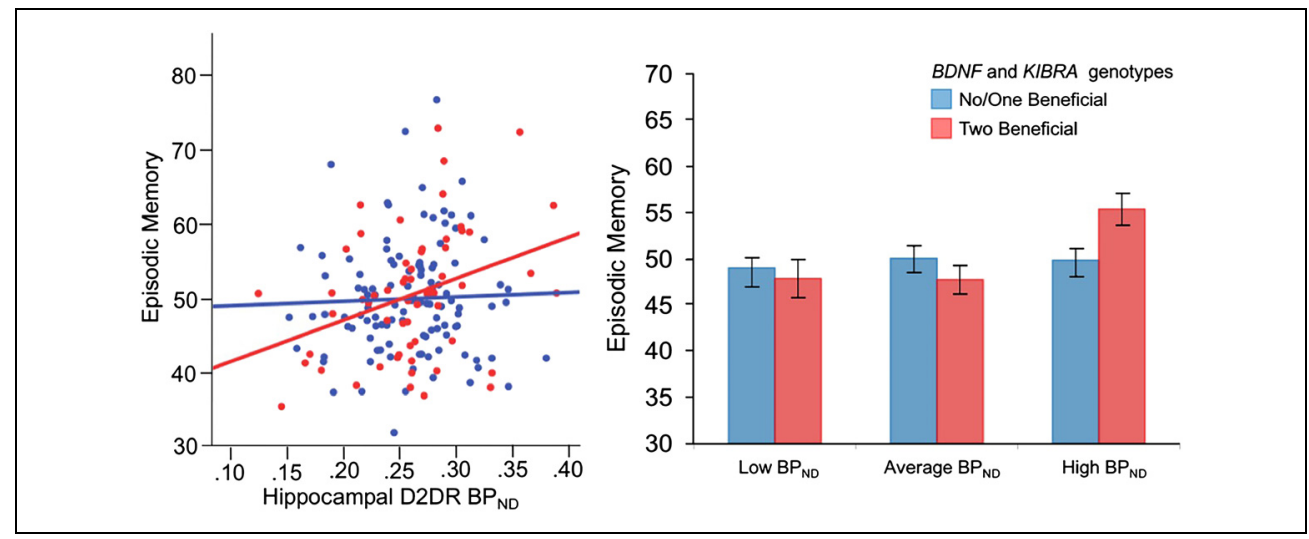

between caudate $\mathrm{BP}_{\mathrm{ND}}$ and episodic memory performance, $\beta=.181, t(168)=2.359, p=.019$, there was no interactive effect between $\mathrm{BP}_{\mathrm{ND}}$ in caudate and the gene score for memory performance $(p=.91)$.

Notably, adjusting the analyses for gray-matter volume in the hippocampus, the pattern of results remained (interaction between hippocampal BPND and the gene score grouping: $\beta=.933, t(165)=2.019, p=.045)$, suggesting that partial volume effects were negligible (estimate for gray matter volume: $\beta=.078, t(165)=0.980$, $p=.329)$.

Figure 1 depicts episodic memory performance as a function of hippocampal D2DR availability (low, average, high) and individual differences in KIBRA and BDNF (one or no beneficial genotype, two beneficial genotypes). Follow-up comparisons indicated that carriers of two advantageous genotypes for KIBRA and BDNF along with high D2DR availability performed better than those with low, $t(35)=3.26, p=.001, \eta_{\mathrm{p}}^{2}=.060$, and average D2DR availability, $t(42)=3.42, p=.001, \eta_{\mathrm{p}}^{2}=.066$. Moreover, the two gene score groups differed significantly in the high D2DR group, $t(55)=2.41, p=.017, \eta_{\mathrm{p}}^{2}=.034$. By contrast, there were no differences among carriers of disadvantageous genotypes across the three D2DR groups $(p s>3)$.

Table 1 shows that advantageous genotypes of KIBRA and $B D N F$ in combination with high hippocampal D2DRs is associated with higher education. There were no other significant differences for this particular group.

\section{DISCUSSION}

We investigated whether hippocampal D2DR availability interacts with memory-related genes (KIBRA, BDNF) to influence episodic memory performance. We show that only carriers of both beneficial genotypes also benefit from higher D2DR availability. Moreover, the subgroup of individuals characterized by high D2DR availability and advantageous genetic predispositions had received more education. The combination of receptor imaging and genetics enables us to specifically focus on hippocampusrelated molecular mechanisms. Although KIBRA and
$B D N F$ are strongly associated with the hippocampus, they are also expressed in other memory-related brain regions, such as cortex and caudate (Johannsen et al., 2008; Hofer et al., 1990). Toward this end, the caudatecognition link was not modulated by KIBRA and BDNF, demonstrating discriminant validity of the current data.

With respect to DA, our previously reported results are in line with its proposed role for episodic memory formation and consolidation (Nyberg et al., 2016). Lisman and Grace (2005) proposed that the ventral tegmental area and the hippocampus form a functional loop, which controls the formation of long-term memories. According to this model, the hippocampus detects a novelty signal. This signal is sent to the ventral tegmental area, where it enhances novelty-related activity of dopaminergic neurons. Consequently, DA is released in the hippocampus, resulting in an enhancement of plasticity-related molecular mechanisms, such as LTP. Our data suggest that DA's role in the hippocampus is closely linked to BDNF and KIBRA. Conceivably, dopaminergic neuromodulation and secretion of KIBRA and BDNF proteins influence episodic memory through the protein kinase Mzeta (PKMzeta), which is involved in synaptic tagging and capture (Sajikumar \& Korte, 2011), creating the potential for a lasting change in synaptic efficacy (Redondo \& Morris, 2011). BDNF facilitates LTP maintenance through PKMzeta (Mei et al., 2011). With respect to KIBRA, it has been shown that this protein also regulates learning and memory through stabilization of PKMzeta (Vogt-Eisele et al., 2014). Animal data indicate that PKMzeta is also critical to the maintenance of episodic memories (for a review, see Glanzman, 2013) and for the induction and maintenance of DA-induced LTP in the hippocampus (Navakkode et al., 2010).

Functional neuroimaging studies have shown that KIBRA influences hippocampal activity both during encoding and retrieval (Muse et al., 2014; Kauppi, Nilsson, Adolfsson, Eriksson, \& Nyberg, 2011; Papassotiropoulos et al., 2006). However, the pattern is less clear for $B D N F$. One study failed to find genotype effects during retrieval processes (Kauppi et al., 2013). By contrast, another study found differences in hippocampal activation 
during both episodic encoding and retrieval tasks (Dennis et al., 2011). Thus, functional effects in the brain of $B D N F$ may be particularly likely to occur during encoding of episodic memories.

The fact that the D2-cognition link was driven by carries of advantageous genotypes is in line with the polygenic nature of complex cognitive functions, such as episodic memory. The pattern of results illustrates that individuals may exhibit poor performance due to different reasons, including low hippocampal D2DR availability and low expression of plasticity-related proteins (KIBRA, BDNF). Accordingly, our results contribute to a better characterization of origins of memory deficits in aging.

The genetic effect in individuals with high D2DR availability is unusually large. Typically, very small effects sizes are common in behavior genetic studies (i.e., less than $1 \%$ of explained variance; for a review, see Plomin, DeFries, Knopik, \& Neiderhiser, 2016). Apart from the genotype effect, PET studies investigating DA-cognition links have not always been able to reveal significant association or found even mixed results (see Juarez et al., 2018, for a review and discussion). Our study emphasizes that it is necessary to consider both PET as well as genetic data to account for individual differences in performance and that some of the mixed results from PET studies may be related to genetic effects. However, future studies will need to replicate the observed interactive effect in independent samples and confirm the relatively large effect size estimate.

The fact that individuals with high hippocampal D2DR availability and advantageous genetic predispositions also had higher education is noteworthy. Although the crosssectional nature of the study does not allow any causal inferences, it is conceivable that carriers of advantageous alleles, along with high D2DR availability, are more likely to have higher educational attainment. Higher education may also be particularly stimulating for individuals with certain genetic predispositions, hence further enhancing genetic expression of KIBRA/BDNF as well as D2DRs via epigenetic mechanisms. This line of reasoning is consistent with animal data demonstrating that environmental enrichment is associated with increased transcription of proteins and alteration of epigenetic markers in the hippocampus (Zhang et al., 2018). Thus, the current pattern may represent a biological pathway through which education exerts beneficial effects on episodic memory (Rönnlund, Nyberg, Bäckman, \& Nilsson, 2005). However, longitudinal data are required to investigate to which extent educational attainment is a mediator or a potential confounding variable of the observed relationship.

Our study sheds new light on the interactive effects of molecular mechanisms in the hippocampus contributing to episodic memory performance in old age. As such, it also contributes to the understanding of the different sources of low episodic memory performance in aging.
Reprint requests should be sent to Goran Papenberg, Aging Research Center, Karolinska Institutet, Tomtebodavägen 18A, 17177 Stockholm, Sweden, or via e-mail: goran.papenberg@ki.se.

\section{REFERENCES}

Ashburner, J., Barnes, G., Chen, C.-C., Daunizeau, J., Flandin, G., Friston, K., et al. (2013). SPM8 manual. Wellcome Trust Centre for Neuroimaging, Institute of Neurology, University College London, London.

Bettinardi, V., Presotto, L., Rapisarda, E., Picchio, M., Gianolli, L., \& Gilardi, M. C. (2011). Physical performance of the new hybrid PET/CT Discovery-690. Medical Physics, 38, 5394-5411.

Binder, D. K., \& Scharfman, H. E. (2004). Brain-derived neurotrophic factor. Growth Factors, 22, 123-131.

Camps, M., Cortés, R., Gueye, B., Probst, A., \& Palacios, J. M. (1989). Dopamine receptors in human brain: Autoradiographic distribution of $\mathrm{D}_{2}$ sites. Neuroscience, 28, 275-290.

Carlsson, A. C., Wändell, P. E., de Faire, U., \& Hellénius, M.-L. (2008). Prevalence of hypertension in immigrants and Swedish-born individuals, a cross-sectional study of 60-yearold men and women in Sweden. Journal of Hypertension, 26, 2295-2302.

Cooke, S. F., \& Bliss, T. V. P. (2006). Plasticity in the human central nervous system. Brain, 129, 1659-1673.

Dennis, N. A., Cabeza, R., Need, A. C., Waters-Metenier, S., Goldstein, D. B., \& LaBar, K. S. (2011). Brain-derived neurotrophic factor val66met polymorphism and hippocampal activation during episodic encoding and retrieval tasks. Hippocampus, 21, 980-989.

de Quervain, D. J.-F., \& Papassotiropoulos, A. (2006). Identification of a genetic cluster influencing memory performance and hippocampal activity in humans. Proceedings of the National Academy of Sciences, U.S.A., 103, 4270-4274.

Egan, M. F., Kojima, M., Callicott, J. H., Goldberg, T. E., Kolachana, B. S., Bertolino, A., et al. (2003). The BDNF val66met polymorphism affects activity-dependent secretion of BDNF and human memory and hippocampal function. Cell, 112, 257-269.

Farde, L., Hall, H., Ehrin, E., \& Sedvall, G. (1986). Quantitative analysis of D2 dopamine receptor binding in the living human brain by PET. Science, 231, 258-261.

Fischl, B., Salat, D. H., Busa, E., Albert, M., Dieterich, M., Haselgrove, C., et al. (2002). Whole brain segmentation: Automated labeling of neuroanatomical structures in the human brain. Neuron, 33, 341-355.

Fischl, B., Salat, D. H., van der Kouwe, A. J. W., Makris, N., Ségonne, F., Quinn, B. T., et al. (2004). Sequenceindependent segmentation of magnetic resonance images. Neuroimage, 23(Suppl. 1), S69-S84.

Frey, U., Huang, Y. Y., \& Kandel, E. R. (1993). Effects of cAMP simulate a late stage of LTP in hippocampal CA1 neurons. Science, 260, 1661-1664.

Frey, U., Schroeder, H., \& Matthies, H. (1990). Dopaminergic antagonists prevent long-term maintenance of posttetanic LTP in the CA1 region of rat hippocampal slices. Brain Research, 522, 69-75.

Glanzman, D. L. (2013). PKM and the maintenance of memory. F1000 Biology Reports, 5, 4.

Han, X., \& Fischl, B. (2007). Atlas renormalization for improved brain MR image segmentation across scanner platforms. IEEE Transactions on Medical Imaging, 26, 479-486.

Herlitz, A., Nilsson, L.-G., \& Bäckman, L. (1997). Gender differences in episodic memory. Memory \& Cognition, 25, 801-811. 
Hirvonen, M. M., Laakso, A., Någren, K., Rinne, J. O., Pohjalainen, T., \& Hietala, J. (2009). C957T polymorphism of dopamine D2 receptor gene affects striatal DRD2 in vivo availability by changing the receptor affinity. Synapse, 63, 907-912.

Hoaglin, D. C., \& Iglewicz, B. (1987). Fine-tuning some resistant rules for outlier labeling. Journal of the American Statistical Association, 82, 1147-1149.

Hofer, M., Pagliusi, S. R., Hohn, A., Leibrock, J., \& Barde, Y. A. (1990). Regional distribution of brain-derived neurotrophic factor mRNA in the adult mouse brain. EMBO Journal, 9, 2459-2464.

Innis, R. B., Cunningham, V. J., Delforge, J., Fujita, M., Gjedde, A., Gunn, R. N., et al. (2007). Consensus nomenclature for in vivo imaging of reversibly binding radioligands. Journal of Cerebral Blood Flow and Metabolism, 27, 1533-1539.

Johannsen, S., Duning, K., Pavenstädt, H., Kremerskothen, J., \& Boeckers, T. M. (2008). Temporal-spatial expression and novel biochemical properties of the memory-related protein KIBRA. Neuroscience, 155, 1165-1173.

Juarez, E. J., Castrellon, J. J., Green, M. A., Crawford, J. L., Seaman, K. L., Smith, C. T., et al. (2018). Reliability of the correlative triad among aging, dopamine D2-like receptor availability, and cognition. bioRxiv, 494765.

Kambeitz, J. P., Bhattacharyya, S., Kambeitz-Ilankovic, L. M., Valli, I., Collier, D. A., \& McGuire, P. (2012). Effect of BDNF val66met polymorphism on declarative memory and its neural substrate: A meta-analysis. Neuroscience $\varepsilon$ Biobehavioral Reviews, 36, 2165-2177.

Karalija, N., Papenberg, G., Wåhlin, A., Johansson, J., Andersson, M., Axelsson, J., et al. (2019). C957T-mediated variation in ligand affinity affects the association between ${ }^{11} \mathrm{C}$-raclopride binding potential and cognition. Journal of Cognitive Neuroscience, 31, 314-325.

Kauppi, K., Nilsson, L.-G., Adolfsson, R., Eriksson, E., \& Nyberg, L. (2011). KIBRA polymorphism is related to enhanced memory and elevated hippocampal processing. Journal of Neuroscience, 31, 14218-14222.

Kauppi, K., Nilsson, L.-G., Adolfsson, R., Lundquist, A., Eriksson, E., \& Nyberg, L. (2013). Decreased medial temporal lobe activation in BDNF ${ }^{66}$ Met allele carriers during memory encoding. Neuropsychologia, 51 , 2462-2468.

Levey, A. I., Hersch, S. M., Rye, D. B., Sunahara, R. K., Niznik, H. B., Kitt, C. A., et al. (1993). Localization of D1 and D2 dopamine receptors in brain with subtype-specific antibodies. Proceedings of the National Academy of Sciences, U.S.A., 90, 8861-8865.

Lisman, J. E., \& Grace, A. A. (2005). The hippocampal-VTA loop: Controlling the entry of information into long-term memory. Neuron, 46, 703-713.

Logan, J., Fowler, J. S., Volkow, N. D., Wang, G.-J., Ding, Y.-S., \& Alexoff, D. L. (1996). Distribution volume ratios without blood sampling from graphical analysis of PET data. Journal of Cerebral Blood Flow and Metabolism, 16, 834-840

Maitland, S. B., Herlitz, A., Nyberg, L., Bäckman, L., \& Nilsson, L.-G. (2004). Selective sex differences in declarative memory. Memory \& Cognition, 32, 1160-1169.

Mei, F., Nagappan, G., Ke, Y., Sacktor, T. C., \& Lu, B. (2011). BDNF facilitates L-LTP maintenance in the absence of protein synthesis through PKM $\zeta$. PLoS One, 6, e21568.

Milnik, A., Heck, A., Vogler, C., Heinze, H.-J., de Quervain, D. J.-F., \& Papassotiropoulos, A. (2012). Association of KIBRA with episodic and working memory: A meta-analysis. American Journal of Medical Genetics, Part B: Neuropsychiatric Genetics, 159B, 958-969.
Mintun, M. A., Raichle, M. E., Kilbourn, M. R., Wooten, G. F., \& Welch, M. J. (1984). A quantitative model for the in vivo assessment of drug binding sites with positron emission tomography. Annals of Neurology, 15, 217-227.

Muse, J., Emery, M., Sambataro, F., Lemaitre, H., Tan, H.-Y., Chen, Q., et al. (2014). WWC1 genotype modulates agerelated decline in episodic memory function across the adult life span. Biological Psychiatry, 75, 693-700.

Navakkode, S., Sajikumar, S., Sacktor, T. C., \& Frey, J. U. (2010). Protein kinase $M \zeta$ is essential for the induction and maintenance of dopamine-induced long-term potentiation in apical CA1 dendrites. Learning \& Memory, 17, 605-611.

Nevalainen, N., Riklund, K., Andersson, M., Axelsson, J., Ögren, M., Lövdén, M., et al. (2015). COBRA: A prospective multimodal imaging study of dopamine, brain structure and function, and cognition. Brain Research, 1612, 83-103.

Nyberg, L., Karalija, N., Salami, A., Andersson, M., Wåhlin, A., Kaboovand, N., et al. (2016). Dopamine D2 receptor availability is linked to hippocampal-caudate functional connectivity and episodic memory. Proceedings of the National Academy of Sciences, U.S.A., 113, 7918-7923.

Nyberg, L., Lövdén, M., Riklund, K., Lindenberger, U., \& Bäckman, L. (2012). Memory aging and brain maintenance. Trends in Cognitive Sciences, 16, 292-305.

Papassotiropoulos, A., \& de Quervain, D. J.-F. (2011). Genetics of human episodic memory: Dealing with complexity. Trends in Cognitive Sciences, 15, 381-387.

Papassotiropoulos, A., Stephan, D. A., Huentelman, M. J., Hoerndli, F. J., Craig, D. W., Pearson, J. V., et al. (2006). Common KIBRA alleles are associated with human memory performance. Science, 314, 475-478.

Papenberg, G., Lindenberger, U., \& Bäckman, L. (2015) Aging-related magnification of genetic effects on cognitive and brain integrity. Trends in Cognitive Sciences, 19, 506-514.

Plomin, R., DeFries, J. C., Knopik, V. S., \& Neiderhiser, J. M. (2016). Top 10 replicated findings from behavioral genetics. Perspectives on Psychological Science, 11, $3-23$.

Rasch, B., Papassotiropoulos, A., \& de Quervain, D. J.-F. (2010). Imaging genetics of cognitive functions: Focus on episodic memory. Neuroimage, 53, 870-877.

Redondo, R. L., \& Morris, R. G. M. (2011). Making memories last: The synaptic tagging and capture hypothesis. Nature Reviews Neuroscience, 12, 17-30.

Rönnlund, M., Nyberg, L., Bäckman, L., \& Nilsson, L.-G. (2005). Stability, growth, and decline in adult life span development of declarative memory: Cross-sectional and longitudinal data from a population-based study. Psychology and Aging, 20, 3-18.

Sajikumar, S., \& Korte, M. (2011). Metaplasticity governs compartmentalization of synaptic tagging and capture through brain-derived neurotrophic factor (BDNF) and protein kinase $\mathrm{M} \zeta(\mathrm{PKM} \zeta)$. Proceedings of the National Academy of Sciences, U.S.A., 108, 2551-2556.

Schneider, A., Huentelman, M. J., Kremerskothen, J., Duning, K., Spoelgen, R., \& Nikolich, K. (2010). KIBRA: A new gateway to learning and memory? Frontiers in Aging Neuroscience, 2, 4

Tabachnick, B. G., \& Fidell, L. S. (2007). Using multivariate statistics (5th ed.). Boston: Allyn \& Bacon/Pearson Education.

Takahashi, H., Kato, M., Hayashi, M., Okubo, Y., Takano, A., Ito, H., et al. (2007). Memory and frontal lobe functions; 
possible relations with dopamine $\mathrm{D} 2$ receptors in the hippocampus. Neuroimage, 34, 1643-1649.

Takahashi, H., Kato, M., Takano, H., Arakawa, R., Okumura, M., Otsuka, T., et al. (2008). Differential contributions of prefrontal and hippocampal dopamine $\mathrm{D}_{1}$ and $\mathrm{D}_{2}$ receptors in human cognitive functions. Journal of Neuroscience, 28, 12032-12038.

Vogt-Eisele, A., Krüger, C., Duning, K., Weber, D., Spoelgen, R., Pitzer, C., et al. (2014). KIBRA (KIdney/BRAin protein) regulates learning and memory and stabilizes protein kinase

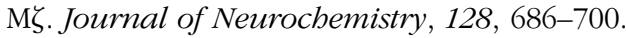

Wallstén, E., Axelsson, J., Sundström, T., Riklund, K., \& Larsson, A. (2013). Subcentimeter tumor lesion delineation for highresolution ${ }^{18}$ F-FDG PET images: Optimizing correction for partial-volume effects. Journal of Nuclear Medicine Technology, 41, 85-91.

Zhang, T.-Y., Keown, C. L., Wen, X., Li, J., Vousden, D. A., Anacker, C., et al. (2018). Environmental enrichment increases transcriptional and epigenetic differentiation between mouse dorsal and ventral dentate gyrus. Nature Communications, 9, 298. 